\title{
A consensus-based template for documenting and reporting in physician-staffed pre-hospital services
}

\author{
Andreas J Krüger ${ }^{1,2,3^{*}}$, David Lockey ${ }^{4}$, Jouni Kurola ${ }^{5}$, Stefano Di Bartolomeo ${ }^{6}$, Maaret Castrén ${ }^{7}$, Søren Mikkelsen ${ }^{8}$ \\ and Hans Morten Lossius ${ }^{1,9}$
}

\begin{abstract}
Background: Physician-staffed pre-hospital units are employed in many Western emergency medical services (EMS) systems. Although these services usually integrate well within their EMS, little is known about the quality of care delivered, the precision of dispatch, and whether the services deliver a higher quality of care to pre-hospital patients. There is no common data set collected to document the activity of physician pre-hospital activity which makes shared research efforts difficult. The aim of this study was to develop a core data set for routine documentation and reporting in physician-staffed pre-hospital services in Europe.

Methods: Using predefined criteria, we recruited sixteen European experts in the field of pre-hospital care. These experts were guided through a four-step modified nominal group technique. The process was carried out using both e-mail-based communication and a plenary meeting in Stavanger, Norway.

Results: The core data set was divided into 5 sections: "fixed system variables", "event operational descriptors", " patient descriptors", "process mapping", and "outcome measures and quality indicators". After the initial round, a total of 361 variables were proposed by the experts. Subsequent rounds reduced the number of core variables to 45. These constituted the final core data set. Emphasis was placed on the standardisation of reporting time variables, chief complaints and diagnostic and therapeutic procedures.
\end{abstract}

Conclusions: Using a modified nominal group technique, we have established a core data set for documenting and reporting in physician-staffed pre-hospital services. We believe that this template could facilitate future studies within the field and facilitate standardised reporting and future shared research efforts in advanced prehospital care.

\section{Background}

Pre-hospital emergency medicine aims to provide early, high-quality, goal-directed treatment. The time to "restoration of adequate flow and physiology" is recognised as crucial in several conditions, including cardiac arrest, cardiac ischaemia, severe trauma, sepsis, respiratory distress, stroke and obstetric emergencies [1]. As our knowledge of acute illness and injury evolves, rapid diagnostics and treatment are becoming more important.

\footnotetext{
* Correspondence: andreas.kruger@snla.no

1 Department of Research and Development, Norwegian Air Ambulance Foundation, Drøbak, Norway

Full list of author information is available at the end of the article
}

It has been argued that an appropriately trained physician is able to provide superior treatment compared to other emergency medical care providers, and, based on this assumption, having a physician as an integrated part of pre-hospital emergency medical care will positively affect patient outcomes [2-5]. These services typically consist of rapid and flexible means of transportation. In larger cities, rapid response cars are often available, and helicopters are common in rural areas. These means of transportation [6,7] facilitate rapid access to the patient and can reduce the time to definitive care at appropriate medical facilities.

The role of the physician-staffed pre-hospital services is not solely medical treatment. The physician often

\section{Biomed Central}


takes an active part in patient and resource triage and acts as a medical advisor/supervisor for ambulance crews.

In Scandinavia, physician-staffed pre-hospital services are well-established and commonly manned with anaesthesiologists to deliver critical emergency care. These services are publicly funded and operate 24 hours a day. The means of transportation (helicopter, rapid response car, ambulance) are provided according to logistical needs. The majority of services are well integrated within their local emergency medical systems [6].

Physician-staffed pre-hospital services demand more resources and are more expensive than standard paramedic-staffed services. The evidence base for which patient groups benefit from physician-manned services and which system factors are related to a favourable patient outcome is currently weak [8].

More research is required to evaluate the effect and efficiency of such services[9]. The well-organised and relatively homogeneous systems of Scandinavia are suited for collaborative research projects. Unfortunately, proper documentation and reporting of medical and operational activity is uncommon and the data that is recorded lacks common variables and definitions. This lack of systematisation of documentation within and between countries is nearly generic in an international perspective and makes it difficult to compare services and to conduct systematic searches in the published literature and multicentre-based prospective or registrybased research [10-13].

In several emergency medical fields, shared research efforts based on common reporting templates have proven useful [14-17]. The Utstein Template for documenting and reporting in cardiac arrest is probably the most widely used template and has facilitated a large amount of research [18-21]. The rationale is to document and report data prospectively with a predefined template developed by experts [22-24]. These templates gather data that can be easily and routinely reported, making multicentre-based research feasible $[25,26])$. Considerably less agreement currently exists on the reporting of the effect and efficiency of emergency medical systems. There is no international agreement on the reporting of fundamental variables, such as outcome variables [10]. Research addressing structure, process and outcome quality indicators, and factors associated with increased value is lacking $[27,28]$.

Templates for documenting and reporting data may be developed using multidisciplinary expert panel consensus methods $[27,29,30]$. Expert panel assessments allow a combination of evidence-based knowledge, personal experience, and general insight in the characteristics of the patient cohort assessed or problem addressed [31], and has been the method of choice in the development of previous Utstein templates [24,32-37]

The aim of this project was to develop a common Utstein-like template for documenting and reporting on pre-hospital emergency medical services manned with trained pre-hospital physicians.

\section{Methods}

The current work is the third part of the ScanDoc project, a Scandinavian consortium working for better research infrastructure in advanced pre-hospital care.

We used a modified nominal group technique [31], and defined "physician-staffed pre-hospital unit" as a dedicated unit staffed with a physician with competency in critical emergency care above that of an ordinary oncall general practitioner.

\section{The experts}

The ScanDoc representatives assisted in proposing experts from the Scandinavian countries. Several countries in central Europe operate pre-hospital physician staffed services similar to those in Scandinavia, and, therefore, it was decided to invite representatives from other countries in Europe. As the original purpose of the ScanDoc-project was to standardise reporting from the Scandinavian countries, the project group decided to weight the panel composition toward Scandinavia, resulting in three representatives from Scandinavia per one from another European country. Based on the following inclusion criteria, panel members were recruited from Sweden, Finland, Denmark, Norway, Italy, Germany, Austria, Switzerland, Holland and the United Kingdom.

1. Relevant clinical experience working in a physician pre-hospital service to ensure personal insight into the operative and medical characteristics of advanced prehospital care.

2. Scientific and/or substantial leadership responsibilities in pre-hospital care to ensure competency in research methods and governance of pre-hospital emergency systems.

3. Ability to communicate in English.

The experts were identified using Medline and Google Scholar searches and via the professional network of the project group. Each expert was invited into the consensus process by e-mail or telephone when feasible. Non-responders were reminded three times by email, followed by direct contact from one of the members of the project group. The selected expert panel participated throughout the complete process, and no additional experts were included after initiating the process. 


\section{The modified nominal group process}

The consensus process was arranged in a four-step sequence (details of each stage are described below). Stages 1, 2 and 4 were carried out by email. During stage 3, the panel met in Stavanger, Norway for a 2-day consensus meeting.

Keeping proposals anonymous is of major methodological importance to reduce the influence of "loud-speaking" experts and to facilitate the influence of their "silent-speaking" peers $[31,38]$. The expert panel members were aware of its composition, but anonymity related to proposals was secured until stage 3 .

Stage 1 . The expert panel received a predesigned worksheet by email. Each expert was asked to propose the ten most important variables to be routinely documented for shared research and benchmarking within each of five predefined sections:

1. Fixed system variables

Variables relating to system characteristics concerning how the service is organised, the operational capacities of the service and its integration with the EMS with which it operate.

2. Event operational descriptors

Variables documenting the context of a mission (dispatch) or episodes of use (for services with advisory functions).

3. Patient descriptors

Variables documenting information related to the patient's profile, e.g., age, gender, co-morbidity and type of medical complaint.

4. Process mapping

Variables recording what happened to the patient and how the episodes of care proceeded.

5 . Outcome and quality indicators

Variables relating to patient and/or mission outcomes, as well as measures of quality.

An optional sixth section for proposals of variables that did not fit into one of the predefined sections was provided. The experts were informed that a subsidiary aim of the process was to establish a core data set that was easy to routinely collect and did not require excessive database alterations.

The project organisers recognised the challenge that several reports on how to document and report data in various parts of emergency care have already been published and implemented $[24,32,37,39]$. These templates (e.g., Utstein for cardiac arrest, the Utstein template for major trauma and the Utstein template for the reporting of advanced airway management) contain some common variables with slightly different definitions. The expert panel was supplied these published templates and asked to make the new variables compatible with existing template variables, if feasible.

The proposed variables were returned to the project group by email and systemised. Different variables with identical meaning were combined carefully so as not to interfere with the expert panel's proposals. No single proposed variables were deleted. The variables within each section were ranked according to how many times the variables had been proposed by the different expert panel members.

Stage 2. The revised worksheet containing aggregated results from stage 1 was sent to the expert panel. The panel were then requested to rank the ten most important variables in each section from 10 (most important) to 1 (least important). The variables with no ranking were then removed from the list. The results from this ranking provided the basis for the consensus meeting.

Stage 3. The expert panel gathered in Stavanger, Norway and, during a 2-day meeting, agreed upon a core data set. At this meeting, moderators (DL and HML)) led the experts through the results from stage 2 . The experts were divided into two groups and discussed portions of the preliminary dataset. The experts subsequently presented their discussions in plenum, and variables were discussed, debated and agreed upon. The variables were decided upon on day 1 and were defined and categorised on day 2 . Some variables were not finally defined during the meeting, and the expert panel approved the project group to propose definitions for the remaining variables before stage 4 .

Stage 4. Based on step 3, the final data set, including definitions, was prepared by the project group and submitted to the expert panel for final approval. At this point, no additional variables were accepted, but minor changes related to answer categories and definitions were allowed.

Consensus was defined as agreement on the proposed variables at the consensus meeting among the attending experts. Furthermore, we informed the experts during stage 4 that no response was interpreted as agreement to the final core data set.

\section{Results}

Of the 17 invited experts, 16 expressed willingness to participate. Fifteen experts returned their proposals during stage one and two, and ten experts attended the consensus meeting. The final core data set was sent to all 15 experts.

In stage one, a total of 361 unique variables were proposed by the experts (additional file 1). After the aggregation of variables with similar content by the project group, 162 different variables were returned to the experts in stage 2 (additional file 2). During stage 2, 98 variables were 
rated and included in the preliminary data set discussed at the consensus meeting (additional file 3).

The final core data set consisted of 45 variables (Table 1,2,3,4 and 5) of which seven consisted of the same variable (physiological data) measured at two different time points (first and last values).
After stage 4, six experts returned minor comments related to a small number of the established definitions. A few minor changes were made accordingly. Moreover, some issues raised in this stage may be important for future revision of the data set. A revision of the template is planned in approximately two years.

Table 1 Fixed system variables.

\begin{tabular}{|c|c|c|c|c|c|c|}
\hline $\begin{array}{l}\text { Data } \\
\text { point } \\
\text { number }\end{array}$ & $\begin{array}{l}\text { Data point } \\
\text { name }\end{array}$ & $\begin{array}{l}\text { Field } \\
\text { format }\end{array}$ & $\begin{array}{l}\text { Data point categories } \\
\text { or values }\end{array}$ & Type* & Exact definition of data point & $\begin{array}{l}\text { Reference/ } \\
\text { Comments }\end{array}$ \\
\hline \multirow[t]{4}{*}{1} & $\begin{array}{l}\text { Speciality of } \\
\text { physician }\end{array}$ & $\begin{array}{l}\text { Nominal } \\
\text { (categorical } \\
\text { data) }\end{array}$ & $1=$ Anaesthesiology & $\begin{array}{l}\text { Bullet } \\
\text { list }\end{array}$ & $\begin{array}{l}\text { Specialist/non-specialist is described in } \\
\text { "Description of system" section. Mixed refers to } \\
\text { both anaesthesiology and emergency medicine }\end{array}$ & $\begin{array}{l}\text { Proposed by project } \\
\text { group }\end{array}$ \\
\hline & & & $\begin{array}{l}2=\text { Emergency } \\
\text { medicine }\end{array}$ & & & \\
\hline & & & $3=$ Mixed & & & \\
\hline & & & 4 = Other & & & \\
\hline 2 & Population & $\begin{array}{l}\text { Continuous } \\
\text { (numerical } \\
\text { data) }\end{array}$ & Number & & $\begin{array}{l}\text { Annual number of citizens in area covered by } \\
\text { service. }\end{array}$ & $\begin{array}{l}\text { EED report } \\
\text { (European } \\
\text { Emergency Data } \\
\text { Project) }\end{array}$ \\
\hline 3 & $\begin{array}{l}\text { Geographical } \\
\text { service area } \\
\text { provision }\end{array}$ & & squared $\mathrm{km}$ & & $\begin{array}{l}\text { Area in which the service is planned to operate, } \\
\text { squared } \mathrm{km}\end{array}$ & EED-report \\
\hline 4 & $\begin{array}{l}\text { MD unit hours } \\
\text { per year }\end{array}$ & Continuous & hh:mm & & $\begin{array}{l}\text { Unit hour is defined as the annual sum of hours } \\
\text { the unit is occupied on a mission. } \sum \text { time } \\
\text { (patient arrival at hospital) - alarm time/year }\end{array}$ & EED-report \\
\hline \multirow[t]{4}{*}{5} & $\begin{array}{l}\text { Mode of } \\
\text { transportation } \\
\text { accessible for the } \\
\text { service }\end{array}$ & $\begin{array}{l}\text { Nominal } \\
\text { (categorical } \\
\text { data) }\end{array}$ & $1=$ Ground & $\begin{array}{l}\text { Check } \\
\text { box }\end{array}$ & $\begin{array}{l}\text { Mode of transportation vehicle(s) available to } \\
\text { service on a regular basis. }\end{array}$ & $\begin{array}{l}\text { Abbreviated from } \\
\text { Utstein Trauma } \\
\text { Registry } 2008\end{array}$ \\
\hline & & & $2=$ Rotor-wing & & & \\
\hline & & & $3=$ Fixed-wing & & & \\
\hline & & & 4 = Other & & & \\
\hline \multirow[t]{3}{*}{6} & Operating hours & Ordinal & $1=$ Full time service & $\begin{array}{l}\text { Bullet } \\
\text { list }\end{array}$ & $\begin{array}{l}\text { Full time service is operational all days and } \\
\text { nights }\end{array}$ & $\begin{array}{l}\text { Proposed by project } \\
\text { group }\end{array}$ \\
\hline & & & $2=$ Day time service & & $\begin{array}{l}\text { Part time service is operational only day time } \\
\text { and/or in hours of light }\end{array}$ & \\
\hline & & & $3=$ Other & & & \\
\hline \multirow[t]{3}{*}{7} & Activation criteria & $\begin{array}{l}\text { Nominal } \\
\text { (categorical } \\
\text { data) }\end{array}$ & $1=$ Criteria-based & $\begin{array}{l}\text { Bullet } \\
\text { list }\end{array}$ & $\begin{array}{l}\text { Service is activated in accordance with a pre- } \\
\text { defined set of activation criteria used by } \\
\text { Emergency Medical Communication Centre }\end{array}$ & $\begin{array}{l}\text { Proposed by expert } \\
\text { panel }\end{array}$ \\
\hline & & & $\begin{array}{l}2=\text { Consultation with } \\
\text { physician }\end{array}$ & & $\begin{array}{l}\text { Service is activated only after consultation with } \\
\text { an on-call physician }\end{array}$ & \\
\hline & & & $3=$ Both & & & \\
\hline 8 & $\begin{array}{l}\text { Number of } \\
\text { events per year }\end{array}$ & $\begin{array}{l}\text { Continuous } \\
\text { (numerical } \\
\text { data) }\end{array}$ & Number & & $\begin{array}{l}\text { Events includes dispatches and requests in } \\
\text { which the physician in service is involved when } \\
\text { on call }\end{array}$ & $\begin{array}{l}\text { "Responses/missions" } \\
\text { recoded to "events" } \\
\text { by project group }\end{array}$ \\
\hline \multirow[t]{3}{*}{9} & Dispatch system & Nominal & $\begin{array}{l}1 \text { = Integrated } \\
\text { Emergency Medical } \\
\text { Communication Centre } \\
\text { (EMCC) }\end{array}$ & $\begin{array}{l}\text { Bullet } \\
\text { list }\end{array}$ & $\begin{array}{l}\text { Integrated EMCC includes dispatch centres } \\
\text { coordinating all levels of pre-hospital services }\end{array}$ & $\begin{array}{l}\text { Proposed by expert } \\
\text { panel }\end{array}$ \\
\hline & & & $2=$ Special EMCC & & $\begin{array}{l}\text { Special EMCC includes centres only responsible } \\
\text { for physician-staffed pre-hospital units }\end{array}$ & \\
\hline & & & $3=$ Both & & & \\
\hline
\end{tabular}

* "Bullet list" means only one possible answer. "Check box" means multiple answers possible. hh:mm: hours: minutes 
Table 2 Event operational descriptors.

\begin{tabular}{|c|c|c|c|c|c|c|}
\hline $\begin{array}{l}\text { Data } \\
\text { variable } \\
\text { no. }\end{array}$ & Data point name & $\begin{array}{l}\text { Type of } \\
\text { data }\end{array}$ & $\begin{array}{l}\text { Data point } \\
\text { categories or } \\
\text { values }\end{array}$ & Type & Definition of data variable & Reference/comment \\
\hline $10 a$ & $\begin{array}{l}\text { Call received at } \\
\text { Emergency Medical } \\
\text { Communication } \\
\text { Centre (EMCC) }\end{array}$ & Continuous & hh:mm & & $\begin{array}{l}\text { The time when the alarm call is } \\
\text { answered at the initial EMCC }\end{array}$ & $\begin{array}{l}\text { Utstein Trauma Registry 2008, Utstein } \\
\text { Dispatch } 2008\end{array}$ \\
\hline $10 \mathrm{~b}$ & Unit arrival on scene & Continuous & hh:mm & & $\begin{array}{l}\text { The time when the vehicle stops } \\
\text { at a location as close as possible } \\
\text { to the patient }\end{array}$ & Utstein cardiac arrest, 2004 \\
\hline $10 \mathrm{c}$ & Patient leaving scene & Continuous & hh:mm & & $\begin{array}{l}\text { The time when the patient is } \\
\text { transferred from the original } \\
\text { location or time of death if dead } \\
\text { on scene }\end{array}$ & $\begin{array}{l}\text { ROC Epistry CA 2005, proposed } \\
\text { revision from project organiser: } \\
\text { change "from scene" to "from } \\
\text { original location" }\end{array}$ \\
\hline $10 d$ & $\begin{array}{l}\text { Patient arrival at } \\
\text { hospital }\end{array}$ & & hh:mm & & $\begin{array}{l}\text { The time when the patient is } \\
\text { formally transferred to receiving } \\
\text { medical facility personnel }\end{array}$ & Proposed by project group \\
\hline \multirow[t]{6}{*}{11} & Type of dispatch & Nominal & $\begin{array}{l}1=\text { Primary } \\
\text { medical } \\
\text { mission }\end{array}$ & $\begin{array}{l}\text { Bullet } \\
\text { list }\end{array}$ & $\begin{array}{l}\text { Includes all primary missions other } \\
\text { than trauma (medical, surgical, } \\
\text { paediatric and obstetric) }\end{array}$ & Proposed by expert panel \\
\hline & & & $\begin{array}{l}2=\text { Primary } \\
\text { trauma mission }\end{array}$ & & & \\
\hline & & & $\begin{array}{l}3=\text { Interhospital } \\
\text { transfer mission }\end{array}$ & & & \\
\hline & & & $\begin{array}{l}4=\text { Search and } \\
\text { rescue mission }\end{array}$ & & & \\
\hline & & & $\begin{array}{l}5= \\
\text { Consultation }\end{array}$ & & & \\
\hline & & & 6 = Other & & & \\
\hline \multirow[t]{5}{*}{12} & Type of transportation & Nominal & $\begin{array}{l}1=\text { Ground } \\
\text { ambulance }\end{array}$ & $\begin{array}{l}\text { Check } \\
\text { box }\end{array}$ & $\begin{array}{l}\text { Main type of vehicle used to } \\
\text { transport the patient to definitive } \\
\text { care. }\end{array}$ & $\begin{array}{l}\text { Abbreviated definition according to } \\
\text { Utstein Trauma Registry } 2008\end{array}$ \\
\hline & & & $\begin{array}{l}2=\text { Helicopter } \\
\text { ambulance }\end{array}$ & & & \\
\hline & & & $3=$ Fixed-wing & & & \\
\hline & & & 4 = Other & & & \\
\hline & & & $\begin{array}{l}5=\text { No } \\
\text { transportation }\end{array}$ & & & \\
\hline \multirow[t]{6}{*}{13} & Result of dispatch & Nominal & $\begin{array}{l}1=\text { Patient } \\
\text { attended }\end{array}$ & $\begin{array}{l}\text { Bullet } \\
\text { list }\end{array}$ & $\begin{array}{l}\text { Dispatch means unit alarmed for } \\
\text { mission or request/advice/ } \\
\text { supervision }\end{array}$ & Proposed by expert panel \\
\hline & & & $\begin{array}{l}2=\text { Mission } \\
\text { aborted due to } \\
\text { weather }\end{array}$ & & & \\
\hline & & & $\begin{array}{l}3=\text { Mission } \\
\text { aborted due to } \\
\text { technical } \\
\text { reasons }\end{array}$ & & & \\
\hline & & & $\begin{array}{l}4=\text { Mission } \\
\text { aborted not } \\
\text { required }\end{array}$ & & & \\
\hline & & & $\begin{array}{l}5=\text { Mission } \\
\text { aborted } \\
\text { alternative } \\
\text { tasking }\end{array}$ & & & \\
\hline & & & $\begin{array}{l}6= \\
\text { Supervision/ } \\
\text { advice only }\end{array}$ & & & \\
\hline
\end{tabular}


Table 3 Patient descriptors

\begin{tabular}{|c|c|c|c|c|c|c|}
\hline $\begin{array}{l}\text { Data } \\
\text { variable } \\
\text { No. }\end{array}$ & $\begin{array}{l}\text { Data variable } \\
\text { name }\end{array}$ & $\begin{array}{l}\text { Type of } \\
\text { data }\end{array}$ & $\begin{array}{l}\text { Data variable } \\
\text { categories or } \\
\text { values }\end{array}$ & Type & Definition of data variable & Comments \\
\hline 14 & Age & Continuous & Number & & The patient's age at the time of event & Utstein Trauma Registry \\
\hline \multirow[t]{3}{*}{15} & Gender & Nominal & $1=$ Female & $\begin{array}{l}\text { Bullet } \\
\text { list }\end{array}$ & The patient's gender & Utstein Trauma Registry \\
\hline & & & $2=$ Male & & & \\
\hline & & & $3=$ Unknown & & & \\
\hline \multirow[t]{7}{*}{16} & Co-morbidity & Ordinal & $\begin{array}{l}1=\text { No }(\text { ASA-PS } \\
=1)\end{array}$ & $\begin{array}{l}\text { Bullet } \\
\text { list }\end{array}$ & ASA-PS definition & American Society of Anaesthesiologists \\
\hline & & & $\begin{array}{l}2=\text { Yes (ASA-PS } \\
=2-6)\end{array}$ & & $1=$ A normal healthy patient & \\
\hline & & & $3=$ Unknown & & $2=$ A patient with mild systemic disease & \\
\hline & & & & & $3=$ A patient with severe systemic disease & \\
\hline & & & & & $\begin{array}{l}4=\text { A patient with severe systemic disease that is a } \\
\text { constant threat to life }\end{array}$ & \\
\hline & & & & & $\begin{array}{l}5=\mathrm{A} \text { moribund patient who is not expected to survive } \\
\text { without operation }\end{array}$ & \\
\hline & & & & & $\begin{array}{l}6=A \text { declared brain-dead patient whose organs are } \\
\text { being removed for donor purposes }\end{array}$ & \\
\hline \multirow[t]{10}{*}{17} & $\begin{array}{l}\text { Medical problem } \\
\text { (main reason for } \\
\text { response) }\end{array}$ & Nominal & $\begin{array}{l}1=\text { Cardiac } \\
\text { arrest }\end{array}$ & $\begin{array}{l}\text { Bullet } \\
\text { list }\end{array}$ & $\begin{array}{l}\text { Select the condition most likely to be the patient's true } \\
\text { medical problem }\end{array}$ & $\begin{array}{l}\text { Proposed by project group. Defined according to EED-project and } \\
\text { ScanDoc phase } 1 \mathrm{~b} \text {. The first } 9 \text { categories should include }>95 \% \text { of all } \\
\text { medical conditions present in daily work }\end{array}$ \\
\hline & & & $2=$ Trauma & & & \\
\hline & & & $\begin{array}{l}3=\text { Breathing } \\
\text { difficulties }\end{array}$ & & & \\
\hline & & & $4=$ Chest pain & & & \\
\hline & & & $5=$ Stroke & & & \\
\hline & & & $\begin{array}{l}6=\text { Acute } \\
\text { neurology } \\
\text { excluding stroke }\end{array}$ & & & \\
\hline & & & $\begin{array}{l}7=\text { Psychiatry } \\
\text { including } \\
\text { intoxication }\end{array}$ & & & \\
\hline & & & $\begin{array}{l}8=\text { Obstetrics } \\
\text { and childbirth }\end{array}$ & & & \\
\hline & & & $9=$ Infection & & & \\
\hline & & & $10=$ Other & & & \\
\hline \multirow[t]{3}{*}{18} & $\begin{array}{l}\text { Dominating type } \\
\text { of injury }\end{array}$ & Nominal & $1=$ Blunt & $\begin{array}{l}\text { Bullet } \\
\text { list }\end{array}$ & Indication of the type of injury produced if trauma & Utstein Trauma Registry \\
\hline & & & $2=$ Penetrating & & & \\
\hline & & & $3=$ Unknown & & & \\
\hline 19 a) & $\begin{array}{l}\text { Glasgow Coma } \\
\text { Scale-first }\end{array}$ & Ordinal & Number (3-15) & & $\begin{array}{l}\text { First recorded pre-interventional Glasgow Coma Scale } \\
\text { upon arrival of physician-staffed service }\end{array}$ & \\
\hline b) & $\begin{array}{l}\text { Glasgow Coma } \\
\text { Scale- last }\end{array}$ & & Number & & $\begin{array}{l}\text { Glasgow Coma Scale at end of care or patient hand- } \\
\text { over }\end{array}$ & \\
\hline 20 a) & Heart rate first & Continuous & $\begin{array}{l}\text { Number (per } \\
\text { minute) }\end{array}$ & & $\begin{array}{l}\text { First heart rate per minute measured by physician- } \\
\text { staffed service }\end{array}$ & \\
\hline
\end{tabular}


Table 3 Patient descriptors (Continued)

\begin{tabular}{|c|c|c|c|c|c|}
\hline b) & \multicolumn{2}{|l|}{ Heart rate last } & \multicolumn{2}{|l|}{ Number } & \multirow{2}{*}{$\begin{array}{l}\text { Heart rate per minute at end-of-care or patient hand- } \\
\text { over } \\
\text { First recorded systolic blood pressure measured by } \\
\text { physician-staffed service (measured with } \\
\text { sphygmomanometer, monitor or intra-arterial line). }\end{array}$} \\
\hline 21 a) & $\begin{array}{l}\text { Systolic blood } \\
\text { pressure-first }\end{array}$ & Continuous & Number $(\mathrm{mmHg})$ & & \\
\hline b) & $\begin{array}{l}\text { Systolic blood } \\
\text { pressure-last }\end{array}$ & & Number & & $\begin{array}{l}\text { Systolic blood pressure at end-of-care or patient } \\
\text { handover }\end{array}$ \\
\hline \multirow[t]{5}{*}{22 a) } & $\begin{array}{l}\text { Cardiac rhythm- } \\
\text { first }\end{array}$ & Ordinal & $\begin{array}{l}1=\text { Sinus } \\
\text { rhythm }\end{array}$ & $\begin{array}{l}\text { Bullet } \\
\text { list }\end{array}$ & $\begin{array}{l}\text { First cardiac rhythm interpreted by physician-staffed } \\
\text { service (minimum 3-channel lead) }\end{array}$ \\
\hline & & & $\begin{array}{l}2=S V E S \\
V E S \text { mono }\end{array}$ & & \\
\hline & & & $\begin{array}{l}3=\text { Atrial Fib/ } \\
\text { Flutt, AV-block } \\
\text { gr. II/III, VES } \text { poly }\end{array}$ & & \\
\hline & & & $\begin{array}{l}4=V F, V T \\
\text { Asystole, PEA }\end{array}$ & & \\
\hline & & & $\begin{array}{l}5=\text { Not } \\
\text { recorded }\end{array}$ & & \\
\hline \multirow[t]{5}{*}{ b) } & $\begin{array}{l}\text { Cardiac rhythm- } \\
\text { last }\end{array}$ & & $\begin{array}{l}1=\text { Sinus } \\
\text { rhythm }\end{array}$ & & Cardiac rhythm at end of care or patient hand-over \\
\hline & & & $\begin{array}{l}2=\text { SVES, } \\
\text { VES mono }\end{array}$ & & \\
\hline & & & $\begin{array}{l}3=\text { Atrial Fib/ } \\
\text { Flutt, AV-block } \\
\text { gr. II/III, VES } \text { poly }_{\text {poly }}\end{array}$ & & \\
\hline & & & $\begin{array}{l}4=\mathrm{VF}, \mathrm{VT} \\
\text { Asystole, PEA }\end{array}$ & & \\
\hline & & & $\begin{array}{l}5=\text { Not } \\
\text { recorded }\end{array}$ & & \\
\hline 23 a) & SpO2-first & Continuous & Number (0-100) & & $\begin{array}{l}\text { First recorded oxygen saturation by physician-staffed } \\
\text { service (measured with pulse oxymeter or arterial blood } \\
\text { gas (SaO2)) }\end{array}$ \\
\hline b) & SpO2-last & & Number & & Oxygen saturation at end-of-care or patient hand-over \\
\hline \multirow[t]{3}{*}{24 a) } & Pain-first & Ordinal & 1 = None & $\begin{array}{l}\text { Bullet } \\
\text { list }\end{array}$ & First level of pain assessed by physician-staffed service \\
\hline & & & 2 = Moderate & & \\
\hline & & & $3=$ Severe & & \\
\hline \multirow[t]{3}{*}{ b) } & Pain-last & & 1 = None & & Level of pain at end of care or patient hand-over \\
\hline & & & 2 = Moderate & & \\
\hline & & & $3=$ Severe & & \\
\hline 25 a) & $\begin{array}{l}\text { Respiratory rate- } \\
\text { first }\end{array}$ & Continuous & $\begin{array}{l}\text { Number (per } \\
\text { minute) }\end{array}$ & & $\begin{array}{l}\text { First respiratory rate per minute measured by physician- } \\
\text { staffed service. If mechanically ventilated, document } \\
\text { ventilation rate. }\end{array}$ \\
\hline b) & $\begin{array}{l}\text { Respiratory rate- } \\
\text { last }\end{array}$ & & Number & & Respiratory rate at end of care or patient hand-over \\
\hline
\end{tabular}

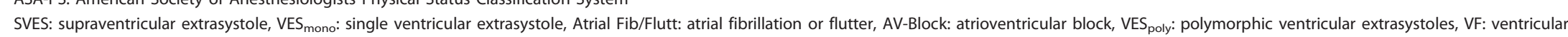
fibrillation, VT: ventricular tachycardia, PEA:pulseless electrical activity 
Table 4 Process mapping.

\begin{tabular}{|c|c|c|c|c|c|c|}
\hline $\begin{array}{l}\text { Data } \\
\text { variable } \\
\text { no. }\end{array}$ & Data variable name & $\begin{array}{l}\text { Type of } \\
\text { data }\end{array}$ & $\begin{array}{l}\text { Data variable } \\
\text { categories or } \\
\text { values }\end{array}$ & Type & Definition of data variable & Comments \\
\hline \multirow[t]{6}{*}{26} & $\begin{array}{l}\text { Diagnostic } \\
\text { procedures }\end{array}$ & Categorical & 1 = US/Doppler & $\begin{array}{l}\text { Check } \\
\text { box }\end{array}$ & & $\begin{array}{l}\text { Proposed } \\
\text { by expert } \\
\text { panel }\end{array}$ \\
\hline & & & $\begin{array}{l}2=\text { ECG- } \\
\text { analysis (12-lead) }\end{array}$ & & & \\
\hline & & & $\begin{array}{l}3=\text { Invasive } \\
\text { monitoring }\end{array}$ & & & \\
\hline & & & $\begin{array}{l}4=\text { Other point- } \\
\text { of-care tests }\end{array}$ & & & \\
\hline & & & $\begin{array}{l}5=\text { Point-of-care } \\
\text { lab tests }\end{array}$ & & & \\
\hline & & & $6=$ Other & & & \\
\hline 27 & $\begin{array}{l}\text { Therapeutic } \\
\text { procedures }\end{array}$ & & & & & \\
\hline \multirow[t]{5}{*}{$27 a$} & $\begin{array}{l}\text { Drugs to facilitate } \\
\text { airway procedure }\end{array}$ & Categorical & $1=$ Sedatives & $\begin{array}{l}\text { Check } \\
\text { box }\end{array}$ & & $\begin{array}{l}\text { Utstein } \\
\text { Airway }\end{array}$ \\
\hline & & & $\begin{array}{l}2= \\
\text { Neuromuscular } \\
\text { blocking agents }\end{array}$ & & & \\
\hline & & & $\begin{array}{l}3=\text { Analgesics/ } \\
\text { opioids }\end{array}$ & & & \\
\hline & & & $\begin{array}{l}4=\text { Local/topic } \\
\text { anaesthetics }\end{array}$ & & & \\
\hline & & & $5=$ None & & & \\
\hline \multirow[t]{7}{*}{$27 \mathrm{~b}$} & $\begin{array}{l}\text { Device used in } \\
\text { successful airway } \\
\text { management }\end{array}$ & Categorical & $\begin{array}{l}1=\text { Bag Mask } \\
\text { Ventilation }\end{array}$ & $\begin{array}{l}\text { Bullet } \\
\text { list }\end{array}$ & $\begin{array}{l}\text { Device used for successful airway management (device in } \\
\text { place at end of care or patient hand-over) }\end{array}$ & $\begin{array}{l}\text { Utstein } \\
\text { Airway }\end{array}$ \\
\hline & & & $2=S A D$ & & & \\
\hline & & & $3=$ Oral TI & & & \\
\hline & & & $4=$ Nasal Tl & & & \\
\hline & & & $\begin{array}{l}5=\text { Surgical } \\
\text { airway }\end{array}$ & & & \\
\hline & & & $6=$ None & & & \\
\hline & & & $7=$ Unknown & & & \\
\hline \multirow[t]{8}{*}{$27 c$} & $\begin{array}{l}\text { Breathing- } \\
\text { procedures used }\end{array}$ & Categorical & $\begin{array}{l}1 \text { = Assisted } \\
\text { manually }\end{array}$ & $\begin{array}{l}\text { Check } \\
\text { box }\end{array}$ & $\begin{array}{l}\text { Open-airway manoeuvres or positioning of patient without } \\
\text { use of any technical airway device (chin-lift, jaw thrust, } \\
\text { recovery position) }\end{array}$ & \\
\hline & & & $\begin{array}{l}2=\text { Assisted } \\
\text { mechanically }\end{array}$ & & $\begin{array}{l}\text { Open-airway manoeuvres, including use of technical devices } \\
\text { (guedel pattern, naso-pharyngeal airway) }\end{array}$ & \\
\hline & & & $\begin{array}{l}3=\text { Controlled } \\
\text { manually }\end{array}$ & & $\begin{array}{l}\text { Breathing assistance using physician's hands (bag-valve-mask } \\
\text { ventilation) }\end{array}$ & \\
\hline & & & $\begin{array}{l}4=\text { Controlled } \\
\text { mechanically }\end{array}$ & & $\begin{array}{l}\text { Breathing assistance using technical respiratory support } \\
\text { (ventilator, NIV) }\end{array}$ & \\
\hline & & & $\begin{array}{l}5=\text { Chest tube/ } \\
\text { decompression }\end{array}$ & & & \\
\hline & & & $\begin{array}{l}6= \\
\text { Thoracostomi }\end{array}$ & & & \\
\hline & & & 7 = Other & & & \\
\hline & & & $8=$ Unknown & & & \\
\hline \multirow[t]{3}{*}{$27 d$} & $\begin{array}{l}\text { Circulation- } \\
\text { procedures used }\end{array}$ & Categorical & $\begin{array}{l}1=\text { Peripheral } \\
\text { IV-line }\end{array}$ & $\begin{array}{l}\text { Check } \\
\text { box }\end{array}$ & & \\
\hline & & & $\begin{array}{l}2=\text { Central IV- } \\
\text { line }\end{array}$ & & & \\
\hline & & & $3=10-$ Access & & & \\
\hline
\end{tabular}




\begin{tabular}{|c|c|c|c|c|c|}
\hline & & & \multicolumn{3}{|l|}{$4=$ Defibrillation } \\
\hline & & & \multicolumn{3}{|l|}{$\begin{array}{l}5= \\
\text { Cardioversion }\end{array}$} \\
\hline & & & \multicolumn{3}{|l|}{$6=$ Pacing } \\
\hline & & & \multicolumn{3}{|l|}{$\begin{array}{l}7= \\
\text { Haemostatic, } \\
\text { basic }\end{array}$} \\
\hline & & & \multicolumn{3}{|l|}{$\begin{array}{l}8= \\
\text { Haemostatic, } \\
\text { advanced }\end{array}$} \\
\hline & & & \multicolumn{3}{|l|}{$9=$ Other } \\
\hline & & & \multicolumn{3}{|l|}{$10=$ None } \\
\hline \multirow[t]{5}{*}{$27 e$} & $\begin{array}{l}\text { Disability- } \\
\text { procedures used }\end{array}$ & Categorical & $\begin{array}{l}1=\text { Reduction } \\
\text { of fractures }\end{array}$ & $\begin{array}{l}\text { Check } \\
\text { box }\end{array}$ & \\
\hline & & & $\begin{array}{l}2 \text { = Spinal } \\
\text { immobilisation }\end{array}$ & & \\
\hline & & & $\begin{array}{l}3=\text { Therapeutic } \\
\text { hypothermia }\end{array}$ & & \\
\hline & & & $4=$ Other & & \\
\hline & & & $5=$ None & & \\
\hline \multirow[t]{2}{*}{28} & $\begin{array}{l}\text { Medication, drugs } \\
\text { administered }\end{array}$ & & $1=$ Yes & $\begin{array}{l}\text { Bullet } \\
\text { list }\end{array}$ & $\begin{array}{l}\text { Indicates whether medication was given by physician-staffed } \\
\text { service. Exclude iv-fluid given for "keep-line-open" purposes }\end{array}$ \\
\hline & & & $2=\mathrm{No}$ & & \\
\hline \multirow[t]{8}{*}{29} & Type of medication & Categorical & $\begin{array}{l}1=\text { Analgesics/ } \\
\text { Opioids }\end{array}$ & $\begin{array}{l}\text { Check } \\
\text { box }\end{array}$ & \\
\hline & & & \multicolumn{3}{|l|}{$2=$ Sedatives } \\
\hline & & & \multicolumn{3}{|l|}{$\begin{array}{l}3=\text { Neuromuscular } \\
\text { blocking agents }\end{array}$} \\
\hline & & & \multicolumn{3}{|l|}{$4=$ Vasoactive } \\
\hline & & & \multicolumn{3}{|l|}{$5=$ Fibrinolytic } \\
\hline & & & \multicolumn{3}{|l|}{$6=$ Antibiotics } \\
\hline & & & \multicolumn{3}{|l|}{7 = Other } \\
\hline & & & \multicolumn{3}{|l|}{8 = None } \\
\hline
\end{tabular}

US: ultrasound

SAD: supraglottic airway device

TI: tracheal intubation

NIV: non invasive ventilation

IO-access: intraosseous access

The "fixed system variables" section contains nine variables, all of which relate to the context of care (Table 1). In the "event operational descriptors" section, seven variables were included derived from four time variables (Table 2). In the third section, "patient descriptors", nineteen variables were decided upon (Table 3). Eight variables were included to describe the diagnostic and therapeutic interventions within the episode of care in the "process mapping" section (Table 4). Finally, 2 variables were included in the "quality Indicator and mission outcome" section (Table 5).

\section{Discussion of core variables}

\section{Background information and fixed system variables}

The expert panel recommended that a description of the system under investigation be reported. These data will typically be "fixed", as they are not patient specific. In cooperative research, these variables can significantly influence comparisons between systems [40-42]. The expert panel divided this section into two parts; one related to the service's context (funding, integration within local EMS, medical capacities, training programs required) and the other including more specific variables, such as medical specialty required for staffing physician, population and service area covered, mode of transportation, operating hours and dispatch system. Strictly defining these variables makes it more straightforward to analyse the influence of system factors.

\section{Event operational descriptors Mission times}

Most physician-manned pre-hospital services document time variables, but there is no international consensus of 
Table 5 Quality indicators and mission outcome.

\begin{tabular}{|c|c|c|c|c|c|c|}
\hline $\begin{array}{l}\text { Data } \\
\text { Variable } \\
\text { No. }\end{array}$ & Data variable name & $\begin{array}{l}\text { Type of } \\
\text { data }\end{array}$ & $\begin{array}{l}\text { Data variable } \\
\text { categories or } \\
\text { values }\end{array}$ & Type & Definition of data variable & Comments \\
\hline 30 & $\begin{array}{l}\text { Physiological improvement from } \\
\text { SpO2, RR, HR, cardiac rhythm, SBP, } \\
\text { GCS and pain }\end{array}$ & & & & $\begin{array}{l}\text { The expert panel recommends developing } \\
\text { Quality Indicators based on changes in } \\
\text { physiological parameters. }\end{array}$ & \\
\hline \multirow[t]{5}{*}{31} & Mission outcome & Nominal & $1=$ Left at scene & $\begin{array}{l}\text { Check } \\
\text { box }\end{array}$ & & $\begin{array}{l}\text { Proposed } \\
\text { by expert } \\
\text { panel }\end{array}$ \\
\hline & & & $\begin{array}{l}2 \text { = Patient to } \\
\text { hospital, not } \\
\text { escorted by } \\
\text { physician }\end{array}$ & & & \\
\hline & & & $\begin{array}{l}3=\text { Patient to } \\
\text { hospital, escorted by } \\
\text { physician }\end{array}$ & & & \\
\hline & & & $\begin{array}{l}4=\text { Declared dead } \\
\text { on arrival at hospital }\end{array}$ & & & \\
\hline & & & $\begin{array}{l}5=\text { Declared dead } \\
\text { at scene }\end{array}$ & & & \\
\hline
\end{tabular}

SpO2: oxygen saturation

RR: respiratory rate per minute

HR: heart rate per minute

SBP: systolic blood pressure

GCS: Glasgow coma scale

the definition of these variables. Since emergency care is often time-critical, standardising how time is documented seems paramount. The expert panel agreed upon documenting time variables as exact times (hh:mm) and not as intervals. Most data registration tools will easily calculate the desired intervals, and it was thought that exact times would provide greater flexibility in statistical handling than intervals.

\section{Call receipt at Emergency Medical Communication Centre (EMCC)}

Response time is commonly documented, and frequently defined as the time from alarm to crew arrival at the scene. This time interval indicates the system's ability to respond to an emergency in a timely manner and is used as a quality indicator in the European Union [43]. The expert panel realised that the receipt of the emergency call at the EMCC is more difficult to collect than the actual alarm time to the attending crew. Nevertheless, the time of receipt of the emergency call from the scene is closer to the actual out-of-hospital time from the patient's perspective, and the expert panel agreed to include this time as the "start of event" indicator.

\section{Type of dispatch}

This indicator variable is important for benchmarking purposes [44], and will not only describe the crude mission profile of the service, but will also be useful for the stratification of mission type data extraction. Some physician-staffed pre-hospital services are dispatched to undifferentiated emergency calls but many are targeted to particular types or severity of incident [6,7]. A substantial workload is imposed onto some services, because of consultation responsibilities for lower-level services (paramedics, dispatch centre) [6]. The expert panel emphasised that this function should also be documented.

\section{Patient descriptors Co-morbidity}

Robust risk-adjustment measures are fundamental for any effort to compare the characteristics of care against hard outcomes [45]. Clearly, the same treatment for the same medical problem can yield different outcomes in groups of patients with different levels of co-morbidity [46]. The expert panel included the well-established and recommended American Society of Anesthesiologists Physical Status Classification System (ASA-PS) [47], albeit in a dichotomised form, as ASA-PS $=1$ or ASA$\mathrm{PS}>1$, as an indicator of co-morbidity. It was considered that while it would be ideal to use the full ASA-scale, obtaining the complete medical history from seriously ill patients is likely to be impossible. It is emphasised that the patient's morbidity prior to the acute medical incident is to be recorded.

\section{Medical problem}

It is crucial for benchmarking to have key variables to serve as inclusion criteria. Because an accurate diagnosis 
is difficult to establish in the pre-hospital setting [48], the expert panel recommended inclusion of a complaint-based indicator for the medical reason for dispatch to better reflect the real-world setting.

\section{First and last values}

As monitoring technology evolves, the amount of vital patient data is almost unlimited, and monitoring strategy is often case-specific. The expert panel recommended documenting these vital data: Glasgow Coma Scale [49], heart rate, systolic blood pressure, cardiac rhythm, oxygen saturation $(\mathrm{SpO} 2)$ and respiratory rate. Typically, such measurements are recorded in a continuous pattern but reporting a complete dynamic profile of the patient's physiology during the process of care will be impractical. The expert panel recommended to report the first and last of these measurements. This approach will form the basis for later analyses of therapeutic and diagnostic interventions related to the patient's clinical condition within the episode of care [50].

\section{Cardiac rhythm}

Advanced anti-arrhythmic treatment is usually confined to physician-staffed pre-hospital services. Several experts noted that recording the cardiac rhythm is often unreliable and not always indicated. Nevertheless, interpretation of cardiac rhythm and treatment of malignant arrhythmias is clearly an advanced intervention. This intervention should be documented, and a four-point scale was accordingly proposed. This scale is inspired from the Mainz Emergency Evaluation Score and separates the cardiac rhythm into normal (sinus rhythm), pathological (atrial fibrillation, AV-block grade 1-2), pathological with circulatory compromise, and cardiac arrest [51]. The expert panel believes that this classification can be applicable using a 3-channel electrocardiogram monitor.

\section{Pain}

Adequate analgesia is regarded as good clinical practice [52], is one major task for pre-hospital services, and should be documented accordingly [53]. There is currently no consensus on which method for documenting pain is most applicable in a pre-hospital setting [54]. Several of the experts argued that the visual analogue scale (VAS) should be used because it is the most established scale in pain assessment. Nevertheless, use of the VAS requires a conscious and cooperative patient, which is not always available in emergency care. A reasonable approach is to document a more coarse scale assessed by the physician. The experts recommend including a three-level pain scale was chosen, consisting of the following classifications: no pain, moderate pain and severe pain.

\section{Process mapping}

Diagnostic procedures were included in an effort to document the specially trained physician's competency in diagnostics. The expert panel agreed that this role in diagnostics will probably be more important in the future.

Therapeutic procedures were systemised according to the traditional $A B C D$ algorithm, and the procedures used in treatment of each were recommended for reporting accordingly.

\section{Medications}

The expert panel agreed upon dividing the medication variable into two types. The first type describes whether any medication was administered, whereas the second documents which type of medication was administered. Emphasis is placed on the type of medication that usually is administered only by physicians. These medications include potent analgesics and sedatives, neuromuscular blocking agents, vasopressors, intravenous antibiotics and anti-arrhythmics.

\section{Quality indicator and outcome measure}

The expert panel suggested the outcome measure should be a description of mission outcome rather than a specific patient medical outcome. Although the recording of long-term patient outcome is ideal it was recognised that this is not achievable in many systems because services often transport patients to different locations and medical facilities.

The expert panel did not agree upon a universal quality indicator during the consensus process. Nevertheless, there seems to be a common opinion that such quality indicators should be developed, and that improvement or deterioration in the clinical condition during the episode of care can serve as a relevant and patient-centred quality indicator. How such a quality indicator should be developed was outside the scope of this consensus process. Several proposed variables can prove useful for selection criteria in future benchmarking and research: variable 11, "type of dispatch", will indicate the main operational indication for dispatch, whereas variable 17 , "medical problem", will further indicate the medical basis for dispatch. When establishing categories within these variables, a compromise between precision and user-friendliness must be achieved; too many categories will be accompanied by difficulties in definitions, whereas too few will result in loss of precision. Therefore, an extended version of the "first-hour quintet" is proposed which [43] will include $>90 \%$ of the medical complaints met in daily service for most services (unpublished data from ScanDoc phase 1b).

\section{General discussion Primary findings}

Using experts within the field and the modified nominal group technique, we defined a core data set to standardise documenting and reporting in physician-staffed pre-hospital care. 
If implemented, we believe that shared research efforts within the field will be enhanced.

The fairly strict definitions and categories within each variable will form the basis for robust documentation and facilitate the extraction of comparable data. The established variables are recommended to reflect ordinary pre-hospital care but also seek to document variables on the specific functions of specially-trained physicians and their exclusive diagnostic and interventional capacities. In addition to being experts in securing vital functions (airway, breathing and circulation), these physicians can apply more aggressive pain control, interpret electrocardiograms and perform advanced diagnostics [8,55-58]. The expert panel agreed on the importance of robust documentation of these advanced interventions, especially those related to the ongoing debate on whether advanced pre-hospital care improves patient outcome.

Shared research efforts require unique patient group identifiers. In the current core data set, several variables can act as such identifiers. Traditionally, pre-hospital treatment strategies have been symptom-based. A diagnostic uncertainty is generally present, and interventions are applied according to the physician's preliminary diagnosis. In the dispatch phase, this diagnostic uncertainty is even more present. Nevertheless, as more advanced diagnostic equipment, such as ultrasound and near patient biochemical tests, are commonly available, diagnostic precision may improve. However since this level of diagnostic equipment is not always accessible, a focus on chief complaints remains fundamental.

\section{Implementation of core data}

The expert panel emphasised that it should be possible to use the core data set without replacing the current infrastructure of documentation. This project does not intend to construct a unique documentation software, as this software could interfere with already developed and wellfunctioning local systems. Therefore, we believe the main benefit of this data set is to standardise current reporting and create increased understanding of how variables should be documented and defined in the future.

\section{Limitations}

Using a consensus methodology to develop a core data set for physician-manned pre-hospital services presents methodological challenges exist [38]. First, there is a risk of selection bias when appointing experts. We believe that the multi-national selection of experts with predefined selection criteria reduced the risk of establishing a biased expert panel. Second, a well-known risk related to the project group's influence on the expert panel is a provision of biased literature before the first round [31]. The project group did provide the expert panel members with a selection of background literature because previously developed and implemented templates were too important to be neglected. Nevertheless, the possibility of this "literature-based" bias seems minimal, given the huge number of variables proposed in the first round.

The specific operational and medical characteristics of the EMS connected to individual experts might have influenced the priorities and proposals during the process. This problem was evident during the consensus meeting, but the panel agreed that the proposed core data set was feasible for most mission types. It is underlined that the current data set represents a minimum, and supplementary variables usually are documented according to service preference.

\section{Summary and conclusions}

Using a modified nominal group technique, we have established an Utstein-like template for documenting and reporting in physician-staffed pre-hospital services. The core data set consists of 45 variables grouped in five different categories. We recommend that future studies in the field report their data according to the template. We believe the template can facilitate future shared research efforts in advanced pre-hospital care.

\section{Additional material}

Additional file 1: Results Stage 1. All proposals from experts after completion of stage 1

Additional file 2: Results Stage 2. All rankings from experts on proposals from stage 1

Additional file 3: Proposals before consensus meeting. All proposals organized by the sum of ranking points from stage 2 .

\section{Acknowledgements}

The project group would like to thank the following for their valuable participation in the expert panel: Ralph Bolander (Sweden), Maaret Castren (Sweden), Erika Christensen (Denmark). Stefano DiBartolomeo (Italy), JonKenneth Heltne (Norway), Jouni Kurola (Finland), Søren Loumann-Nielsen (Denmark), Sindre Mellesmo (Norway), Søren Mikkelsen (Denmark), Anders Nakstad (Norway), Akkie Ringburg (The Netherlands), Tuukka Toivio (Finland), Wolfgang Ummenhofer (Switzerland), Wolfgang Voelckel (Austria) and Mikael Äyras (Finland)

The project was funded by the Laerdal Foundatation for Acute Medicine and Norwegian Air Ambulance Foundation (SNLA). The first author (AJK) holds a position as a PhD-student financed by SNLA.

\section{Author details}

${ }^{1}$ Department of Research and Development, Norwegian Air Ambulance Foundation, Drøbak, Norway. ${ }^{2}$ Department of Anaesthesia and Emergency Medicine, St. Olav University Hospital, Trondheim, Norway. ${ }^{3}$ Department of Circulation and Medical Imaging, Faculty of Medicine, Norwegian University of Science and Technology (NTNU), Trondheim, Norway. ${ }^{4}$ North Bristol NHS trust, Bristol, UK. ${ }^{5}$ Centre for Prehospital Care, division of prehospital emergency care, emergency and intensive care, Kuopio University Hospital, Kuopio, Finland. ${ }^{6}$ Anaesthesia and ICU S.M.M Hospital, Udine/Regional Health Agency of Emilia-Romagna, Bologna, Italy. ${ }^{7}$ Karolinska Institutet, Department of Clinical Science and Education, Södersjukhuset and Section of Emergency 
Medicine, Södersjukhuset, Stockholm, Sweden. ${ }^{8}$ Mobile Emergency Care Unit, Department of Anaesthesiology and Intensive Care Medicine, Odense University Hospital, Odense, Denmark. ${ }^{9}$ Department of Surgical Sciences, University of Bergen, Norway.

\section{Authors' contributions}

AK and HML conceived and organized the study. AK, HML organised, and $D L, H M L$ and SDB moderated the consensus meeting. AK and HML drafted the manuscript, and all authors read and approved the final manuscript.

\section{Competing interests}

The authors declare that they have no competing interests.

Received: 29 September 2011 Accepted: 23 November 2011 Published: 23 November 2011

\section{References}

1. Ghosh R, Pepe P: The critical care cascade: a systems approach. Curr Opin Crit Care 2009, 15:279-283.

2. Lossius HM, Soreide E, Hotvedt R, Hapnes SA, Eielsen OV, Forde $\mathrm{OH}$, Steen PA: Prehospital advanced life support provided by specially trained physicians: is there a benefit in terms of life years gained? Acta Anaesthesiol Scand 2002, 46:771-778.

3. Kurola J, Wangel M, Uusaro A, Ruokonen E: Paramedic helicopter emergency service in rural Finland - do benefits justify the cost? Acta Anaesthesiol Scand 2002, 46:779-784.

4. Frankema SP, Ringburg AN, Steyerberg EW, Edwards MJ, Schipper IB, van Vugt AB: Beneficial effect of helicopter emergency medical services on survival of severely injured patients. Br J Surg 2004, 91:1520-1526.

5. Rehn M, Eken T, Kruger AJ, Steen PA, Skaga NO, Lossius HM: Precision of field triage in patients brought to a trauma centre after introducing trauma team activation guidelines. Scand I Trauma Resusc Emerg Med 2009, 17:1.

6. Kruger AJ, Skogvoll E, Castren M, Kurola J, Lossius HM: Scandinavian prehospital physician-manned Emergency Medical Services-same concept across borders? Resuscitation 2010, 81:427-433

7. Langhelle A, Lossius HM, Silfvast T, Bjornsson HM, Lippert FK, Ersson A, Soreide E: International EMS Systems: the Nordic countries. Resuscitation 2004, 61:9-21

8. Fischer M, Kamp J, Garcia-Castrillo Riesgo L, Robertson-Steel I, Overton J, Ziemann A, Krafft T: Comparing emergency medical service systems-a project of the European Emergency Data (EED) Project. Resuscitation 2011, 82:285-293.

9. Fevang E, Lockey D, Thompson J, Lossius HM: The top five research priorities in physician-provided pre-hospital critical care: a consensus report from a European research collaboration. Scand I Trauma Resusc Emerg Med 2011, 19:57.

10. MacFarlane C: The advances and evidence base for prehospital care. Emerg Med J 2003, 20:114-115.

11. Lossius HM, Sollid SJ, Rehn M, Lockey DJ: Revisiting the value of prehospital tracheal intubation: an all time systematic literature review extracting the Utstein airway core variables. Crit Care 2011, 15:R26.

12. Cone DC: Knowledge translation in the emergency medical services: a research agenda for advancing prehospital care. Acad Emerg Med 2007, 14:1052-1057.

13. Mann NC, Dean JM, Mobasher H, Mears G, Ely M: The use of national highway traffic safety administration uniform prehospital data elements in state emergency medical services data collection systems. Prehosp Emerg Care 2004, 8:29-33.

14. Bulger EM, May S, Kerby JD, Emerson S, Stiell IG, Schreiber MA, Brasel KJ Tisherman SA, Coimbra R, Rizoli S, Minei JP, Hata JS, Sopko G, Evans DC, Hoyt DB: Out-of-hospital hypertonic resuscitation after traumatic hypovolemic shock: a randomized, placebo controlled trial. Ann Surg 2011, 253:431-441.

15. Hostler D, Everson-Stewart S, Rea TD, Stiell IG, Callaway CW, Kudenchuk PJ, Sears GK, Emerson SS, Nichol G: Effect of real-time feedback during cardiopulmonary resuscitation outside hospital: prospective, clusterrandomised trial. Bmj 2011, 342:d512.

16. Aufderheide TP, Nichol G, Rea TD, Brown SP, Leroux BG, Pepe PE, Kudenchuk PJ, Christenson J, Daya MR, Dorian P, Callaway CW, Idris AH, Andrusiek D, Stephens SW, Hostler D, Davis DP, Dunford JV, Pirrallo RG,
Stiell IG, Clement CM, Craig A, Van Ottingham L, Schmidt TA, Wang HE, Weisfeldt ML, Ornato JP, Sopko G: A Trial of an Impedance Threshold Device in Out-of-Hospital Cardiac Arrest. N Engl J Med 2011, 365:798-806.

17. Stiell IG, Nichol G, Leroux BG, Rea TD, Ornato JP, Powell J, Christenson J, Callaway CW, Kudenchuk PJ, Aufderheide TP, Idris AH, Daya MR, Wang HE, Morrison LJ, Davis D, Andrusiek D, Stephens S, Cheskes S, Schmicker RH, Fowler R, Vaillancourt C, Hostler D, Zive D, Pirrallo RG, Vilke GM, Sopko G, Weisfeldt M: Early versus later rhythm analysis in patients with out-ofhospital cardiac arrest. N Engl J Med 2011, 365:787-797.

18. Olasveengen TM, Sunde K, Brunborg C, Thowsen J, Steen PA, Wik L: Intravenous drug administration during out-of-hospital cardiac arrest: a randomized trial. Jama 2009, 302:2222-2229.

19. Stiell IG, Wells GA, Field B, Spaite DW, Nesbitt LP, De Maio VJ, Nichol G, Cousineau D, Blackburn J, Munkley D, Luinstra-Toohey L, Campeau T, Dagnone E, Lyver M: Advanced cardiac life support in out-of-hospital cardiac arrest. The New England journal of medicine 2004, 351:647-656.

20. Weisfeldt ML, Sitlani CM, Ornato JP, Rea T, Aufderheide TP, Davis D, Dreyer J, Hess EP, Jui J, Maloney J, Sopko G, Powell J, Nichol G, Morrison LJ: Survival after application of automatic external defibrillators before arrival of the emergency medical system: evaluation in the resuscitation outcomes consortium population of 21 million. J Am Coll Cardiol 2010, 55:1713-1720.

21. Atkins DL, Everson-Stewart S, Sears GK, Daya M, Osmond MH, Warden CR, Berg RA: Epidemiology and outcomes from out-of-hospital cardiac arrest in children: the Resuscitation Outcomes Consortium Epistry-Cardiac Arrest. Circulation 2009, 119:1484-1491.

22. Jacobs I, Nadkarni V, Bahr J, Berg RA, Billi JE, Bossaert L, Cassan P, Coovadia A, D'Este K, Finn J, Halperin H, Handley A, Herlitz J, Hickey R, Idris A, Kloeck W, Larkin GL, Mancini ME, Mason P, Mears G, Monsieurs K, Montgomery W, Morley P, Nichol G, Nolan J, Okada K, Perlman J, Shuster M, Steen PA, Sterz F, et al: Cardiac arrest and cardiopulmonary resuscitation outcome reports: update and simplification of the Utstein templates for resuscitation registries: a statement for healthcare professionals from a task force of the International Liaison Committee on Resuscitation (American Heart Association, European Resuscitation Council, Australian Resuscitation Council, New Zealand Resuscitation Council, Heart and Stroke Foundation of Canada, InterAmerican Heart Foundation, Resuscitation Councils of Southern Africa). Circulation 2004, 110:3385-3397.

23. Ringdal KG, Coats TJ, Lefering R, Di Bartolomeo S, Steen PA, Roise O, Handolin L, Lossius HM: The Utstein template for uniform reporting of data following major trauma: A joint revision by SCANTEM, TARN, DGUTR and RITG. Scand J Trauma Resusc Emerg Med 2008, 16:7.

24. Sollid SJ, Lockey D, Lossius HM: A consensus-based template for uniform reporting of data from pre-hospital advanced airway management. Scand I Trauma Resusc Emerg Med 2009, 17:58.

25. MacFarlane C, Benn CA: Evaluation of emergency medical services systems: a classification to assist in determination of indicators. Emerg Med J 2003, 20:188-191.

26. Pepe PE, Copass MK, Sopko G: Clinical trials in the out-of-hospital setting: rationale and strategies for successful implementation. Crit Care Med 2009, 37:S91-101.

27. Snooks H, Evans A, Wells B, Peconi J, Thomas M, Woollard M, Guly H, Jenkinson E, Turner J, Hartley-Sharpe C: What are the highest priorities for research in emergency prehospital care? Emerg Med J 2009, 26:549-550.

28. Callaham M: Quantifying the scanty science of prehospital emergency care. Ann Emerg Med 1997, 30:785-790.

29. Maio RF, Garrison HG, Spaite DW, Desmond JS, Gregor MA, Cayten CG, Chew JL, Hill EM, Joyce SM, MacKenzie EJ, Miller DR, O'Malley PJ, Stiell IG: Emergency medical services outcomes project I (EMSOP I): prioritizing conditions for outcomes research. Ann Emerg Med 1999, 33:423-432.

30. Rosengart MR, Nathens AB, Schiff MA: The identification of criteria to evaluate prehospital trauma care using the Delphi technique. J Trauma 2007, 62:708-713.

31. Murphy MK, Black NA, Lamping DL, McKee CM, Sanderson CF, Askham J, Marteau T: Consensus development methods, and their use in clinical guideline development. Health Technol Assess 1998, 2:i-iv, 1-88.

32. Ringdal KG, Coats TJ, Lefering R, Di Bartolomeo S, Steen PA, Roise O, Handolin L, Lossius HM: The Utstein template for uniform reporting of data following major trauma: a joint revision by SCANTEM, TARN, DGUTR and RITG. Scand J Trauma Resusc Emerg Med 2008, 16:7. 
33. Cummins RO, Chamberlain DA, Abramson NS, Allen M, Baskett PJ, Becker $L$, Bossaert L, Delooz HH, Dick WF, Eisenberg MS, et al: Recommended guidelines for uniform reporting of data from out-of-hospital cardiac arrest: the Utstein Style. A statement for health professionals from a task force of the American Heart Association, the European Resuscitation Council, the Heart and Stroke Foundation of Canada, and the Australian Resuscitation Council. Circulation 1991, 84:960-975.

34. Idris AH, Becker LB, Ornato JP, Hedges JR, Bircher NG, Chandra NC, Cummins RO, Dick W, Ebmeyer U, Halperin HR, Hazinski MF, Kerber RE, Kern KB, Safar P, Steen PA, Swindle MM, Tsitlik JE, von Planta I, von Planta M, Wears RL, Weil MH: Utstein-style guidelines for uniform reporting of laboratory CPR research. A statement for healthcare professionals from a Task Force of the American Heart Association, the American College of Emergency Physicians, the American College of Cardiology, the European Resuscitation Council, the Heart and Stroke Foundation of Canada, the Institute of Critical Care Medicine, the Safar Center for Resuscitation Research, and the Society for Academic Emergency Medicine. Resuscitation 1996, 33:69-84

35. Idris AH, Berg RA, Bierens J, Bossaert L, Branche CM, Gabrielli A, Graves SA, Handley AJ, Hoelle R, Morley PT, Papa L, Pepe PE, Quan L, Szpilman D, Wigginton JG, Modell $J H$ : Recommended guidelines for uniform reporting of data from drowning: the "Utstein style". Circulation 2003, 108:2565-2574.

36. Langhelle A, Nolan J, Herlitz J, Castren M, Wenzel V, Soreide E, Engdahl J, Steen PA: Recommended guidelines for reviewing, reporting, and conducting research on post-resuscitation care: the Utstein style. Resuscitation 2005, 66:271-283.

37. Castren M, Karlsten R, Lippert F, Christensen EF, Bovim E, Kvam AM, Robertson-Steel I, Overton J, Kraft T, Engerstrom L, Garcia-Castrill Riego L: Recommended guidelines for reporting on emergency medical dispatch when conducting research in emergency medicine: the Utstein style. Resuscitation 2008, 79:193-197.

38. Sinha IP, Smyth RL, Williamson PR: Using the Delphi technique to determine which outcomes to measure in clinical trials: recommendations for the future based on a systematic review of existing studies. PLoS Med 2011, 8:e1000393.

39. Jacobs I, Nadkarni V, Bahr J, Berg RA, Billi JE, Bossaert L, Cassan P, Coovadia A, D'Este K, Finn J, Halperin H, Handley A, Herlitz J, Hickey R, Idris A, Kloeck W, Larkin GL, Mancini ME, Mason P, Mears G, Monsieurs K, Montgomery W, Morley P, Nichol G, Nolan J, Okada K, Perlman J, Shuster M, Steen PA, Sterz F, et al: Cardiac arrest and cardiopulmonary resuscitation outcome reports: update and simplification of the Utstein templates for resuscitation registries. A statement for healthcare professionals from a task force of the international liaison committee on resuscitation (American Heart Association, European Resuscitation Council, Australian Resuscitation Council, New Zealand Resuscitation Council, Heart and Stroke Foundation of Canada, InterAmerican Heart Foundation, Resuscitation Council of Southern Africa). Resuscitation 2004, 63:233-249.

40. Roudsari BS, Nathens AB, Cameron P, Civil I, Gruen RL, Koepsell TD, Lecky FE, Lefering RL, Liberman M, Mock CN, Oestern HJ, Schildhauer TA, Waydhas C, Rivara FP: International comparison of prehospital trauma care systems. Injury 2007, 38:993-1000.

41. Craig P, Dieppe P, Macintyre S, Michie S, Nazareth I, Petticrew M: Developing and evaluating complex interventions: the new Medical Research Council guidance. Bmj 2008, 337:a1655.

42. Bossaert LL: The complexity of comparing different EMS systems-a survey of EMS systems in Europe. Ann Emerg Med 1993, 22:99-102.

43. European Emergency Data Project. [http://www.geographie.unimuenchen.de/eed/assets/publications/eed_report_titel-index.pdf].

44. Newgard CD, Lewis RJ: Statistical methods for prehospital care research. Prehosp Emerg Care 2002, 6:59-17.

45. Garrison HG, Maio RF, Spaite DW, Desmond JS, Gregor MA, O'Malley PJ, Stiell IG, Cayten CG, Chew JL, Mackenzie EJ, Miller DR: Emergency Medical Services Outcomes Project III (EMSOP III): the role of risk adjustment in out-of-hospital outcomes research. Ann Emerg Med 2002, 40:79-88.

46. Skaga NO, Eken T, Sovik S, Jones JM, Steen PA: Pre-injury ASA physical status classification is an independent predictor of mortality after trauma. J Trauma 2007, 63:972-978.

47. ASA Physical Status Classification System. [http://www.asahq.org/clinical/ physicalstatus.htm]
48. Burman RA, Zakariassen E, Hunskaar S: Acute chest pain - A prospective population based study of contacts to Norwegian emergency medical communication centres. BMC Emerg Med 2011, 11:9.

49. Teasdale $G$, Jennett $B$ : Assessment of coma and impaired consciousness. A practical scale. Lancet 1974, 2:81-84.

50. Grmec S, Kupnik D: Does the Mainz Emergency Evaluation Scoring (MEES) in combination with capnometry (MEESc) help in the prognosis of outcome from cardiopulmonary resuscitation in a prehospital setting? Resuscitation 2003, 58:89-96.

51. Grmec S, Gasparovic V: Comparison of APACHE II, MEES and Glasgow Coma Scale in patients with nontraumatic coma for prediction of mortality. Acute Physiology and Chronic Health Evaluation. Mainz Emergency Evaluation System. Crit Care 2001, 5:19-23.

52. Thomas SH, Shewakramani S: Prehospital trauma analgesia. J Emerg Med 2008, 35:47-57.

53. Maio RF, Garrison HG, Spaite DW, Desmond JS, Gregor MA, Stiell IG, Cayten CG, Chew JL, Mackenzie EJ, Miller DR, PJ OM: Emergency Medical Services Outcomes Project (EMSOP) IV: pain measurement in out-ofhospital outcomes research. Ann Emerg Med 2002, 40:172-179.

54. Jennings PA, Cameron P, Bernard S: Measuring acute pain in the prehospital setting. Emerg Med J 2009, 26:552-555

55. Bakalos G, Mamali M, Komninos C, Koukou E, Tsantilas A, Tzima S, Rosenberg T: Advanced life support versus basic life support in the prehospital setting: A meta-analysis. Resuscitation 2011, 82:1130-1137.

56. Wildner G, Pauker N, Archan S, Gemes G, Rigaud M, Pocivalnik M, Prause G: Arterial line in prehospital emergency settings - A feasibility study in four physician-staffed emergency medical systems. Resuscitation 2011, 82:1198-1201.

57. Rempell JS, Noble VE: Using lung ultrasound to differentiate patients in acute dyspnea in the prehospital emergency setting. Crit Care 2011, $15: 161$.

58. Jabre P, Combes X, Lapostolle F, Dhaouadi M, Ricard-Hibon A, Vivien B, Bertrand L, Beltramini A, Gamand P, Albizzati S, Perdrizet D, Lebail G, Chollet-Xemard C, Maxime V, Brun-Buisson C, Lefrant JY, Bollaert PE, Megarbane B, Ricard JD, Anguel N, Vicaut E, Adnet F: Etomidate versus ketamine for rapid sequence intubation in acutely ill patients: a multicentre randomised controlled trial. Lancet 2009, 374:293-300.

doi:10.1186/1757-7241-19-71

Cite this article as: Krüger et al:: A consensus-based template for documenting and reporting in physician-staffed pre-hospital services. Scandinavian Journal of Trauma, Resuscitation and Emergency Medicine 2011 19:71.

\section{Submit your next manuscript to BioMed Central and take full advantage of:}

- Convenient online submission

- Thorough peer review

- No space constraints or color figure charges

- Immediate publication on acceptance

- Inclusion in PubMed, CAS, Scopus and Google Scholar

- Research which is freely available for redistribution 